\title{
Imaging for cardiac electrophysiology
}

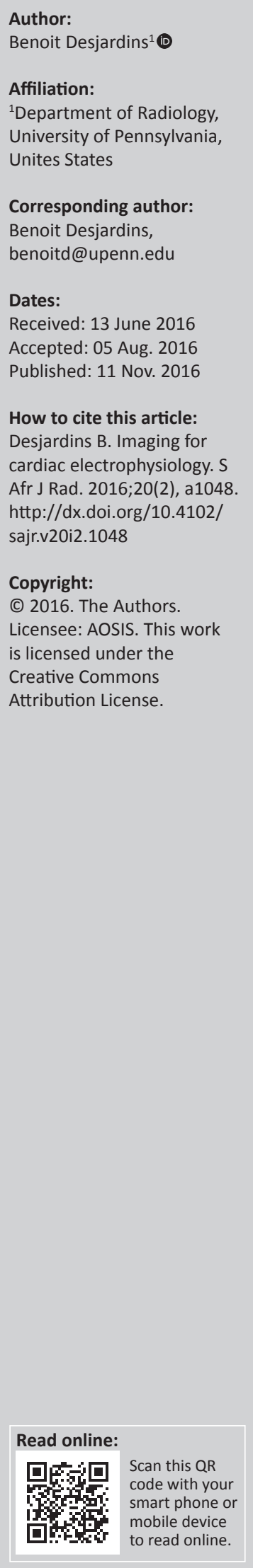

Clinical cardiac electrophysiology is the study of the origin and treatment of arrhythmia. There has been considerable recent development in this field, where imaging has had a transformational impact. In this invited review, we offer a global overview of the most important developments in the use of imaging in cardiac electrophysiology. We first describe the radiological imaging modalities involved in cardiac electrophysiology, to assess cardiac anatomy, function and scar. We then introduce an imaging modality with which readers are probably unfamiliar (electroanatomical mapping [EAM]), but which is routinely used by electrophysiologists to plan and guide cardiac mapping and cardiac ablation therapy by catheter, a therapy which can reduce or even cure arrhythmia. We identify the limitations of EAM and describe how radiological imaging modalities can complement this technique. We then describe and illustrate how imaging has helped the diagnosis of arrhythmogenic conditions, and how imaging is used to plan and guide clinical cardiac electrophysiologic procedures and assess their results and complications. We focus on the two most common arrhythmias for which imaging has the greatest impact: atrial fibrillation and ventricular tachycardia.

\section{Introduction}

Clinical cardiac electrophysiology is the study of the origin and treatment of arrhythmia, which is an abnormality in the rate, regularity or sequence of cardiac activation. Over 2 million people are affected by arrhythmia in South Africa. Some of these arrhythmias (post-infarct arrhythmia) are immediately life threatening. Other arrhythmias (atrial fibrillation [AF]) are not immediately life threatening, but lead to important complications such as strokes.

Imaging has become an integral part of clinical cardiac electrophysiology. It helps to diagnose arrhythmogenic conditions, to plan and guide palliative or potentially curative procedures, and to assess the results and complications of these procedures. An increasingly popular procedure that can improve or cure arrhythmia is cardiac ablation therapy, which uses catheters to create thermal burns on the surface of the myocardium, to destroy arrhythmogenic foci or pathways.

In this invited review, we provide examples of the modern uses of imaging in cardiac electrophysiology. We first introduce the different imaging modalities used in cardiac electrophysiology, focusing on their integration, and discuss how they are used in the context of $\mathrm{AF}$ and ventricular tachycardia (Table 1$)$.

\section{Imaging tools}

Several radiological imaging modalities are used in cardiac electrophysiology, including plain film, computed tomography (CT), magnetic resonance imaging (MRI), single-photon emission computed tomography (SPECT), positron emission tomography (PET), angiography and ultrasound. Of these, CT, MRI and ultrasound are most closely integrated into clinical systems used in cardiac electrophysiology. These imaging modalities are not only able to assess anatomy and function, but can also assess myocardial scar, which is of crucial importance in cardiac electrophysiology.

\section{Imaging of anatomy and function}

Although CT and MRI can assess both anatomy and function, functional imaging from these modalities has not yet been integrated into cardiac electrophysiology systems, so we will focus on the acquisition of high-quality static imaging by CT or MRI. Imaging of patients with arrhythmia is not easy, and incorrect techniques lead to blurry images. AF and premature ventricular contractions (PVCs) are the two most common arrhythmias affecting image acquisition.

Cardiac computed tomography angiography (CTA) can be performed with or without ECG gating. Imaging with cardiac gating is preferred, as it produces images that are not corrupted by cardiac motion. 
However, if a patient has an arrhythmia during the acquisition of gated images, this will create imaging artifacts. This problem can be addressed by using retrospective gating followed by ECGediting of the CTA dataset, which can correct corrupted imaging data from a few PVCs, or by using high pitch techniques, which can acquire images in patients with AF (Figure 1a). Cardiac magnetic resonance angiography (MRA) can be performed using a first-pass intravenous (IV) contrast MRA technique, using arrhythmia rejection algorithms on the scanner. A free-breathing

TABLE 1: Role of imaging modalities.

\begin{tabular}{lll}
\hline Variable & Atrial ablation & Ventricular ablation \\
\hline Planning & LA and PV anatomy $\dagger$ & LV anatomy $\dagger$ \\
& Thrombus $\dagger$ & LV scar $\dagger$ \\
& Surrounding structures $\dagger$ & Coronary veins and arteries $\dagger$ \\
& LA scar $\dagger$ & Epicardial fat $\dagger$ \\
& - & Denervation (SPECT, PET) $\dagger$ \\
Guidance & Catheter navigation $\dagger$ & Anatomy $\dagger$ \\
& Surrounding structures $\dagger$ & Thrombus $\dagger$ \\
& Location of ablation $\dagger$ & Papillary muscles $\dagger$ \\
& Complications $\dagger$ & Real-time guidance \\
Post procedure & Complications $\dagger$ & Complications $\dagger$ \\
& Ablation lesions $\dagger$ & Ablation lesions $\dagger$ \\
\hline
\end{tabular}

LA, left atrium; PV, pulmonary vein; SPECT, single-photon emission computed tomography; $\mathrm{PET}$, positron emission tomography.

$\dagger, \mathrm{CT}, \mathrm{MRI} ; \uparrow, \mathrm{CT}, \mathrm{MRI}$, Intracardiac echocardiography. whole heart MRI technique is more robust, as it acquires MRI data over a longer period of time (10 $\mathrm{min}-15 \mathrm{~min})$, and can reject all bad data acquired during arrhythmia (Figure 1b).

\section{Imaging of scar}

Myocardial scar is intimately related to the generation of arrhythmia, and therefore visualisation of scar is one of the main foci in imaging for cardiac electrophysiology. Scar imaging by late gadolinium enhancement (LGE) MRI has become the gold standard. After an IV bolus of gadoliniumbased contrast agent, myocardial imaging is performed after a delay of $10 \mathrm{~min}-15 \mathrm{~min}$. After such a delay, the contrast agent has mostly cleared from normal myocardium, but areas of scar have increased extra-cellular spaces and retain the contrast agent longer, creating a difference in signal intensity. This difference is further amplified by the use of nulling pulses. Figure 2a shows areas of subendocardial and transmural scar in ischaemic cardiomyopathy, and Figure $2 b$ shows patchy scar in cardiac sarcoid.

Another approach to image myocardial scar, which is less known to radiologists, is electroanatomical mapping (EAM) by catheter. This technique has been used to measure

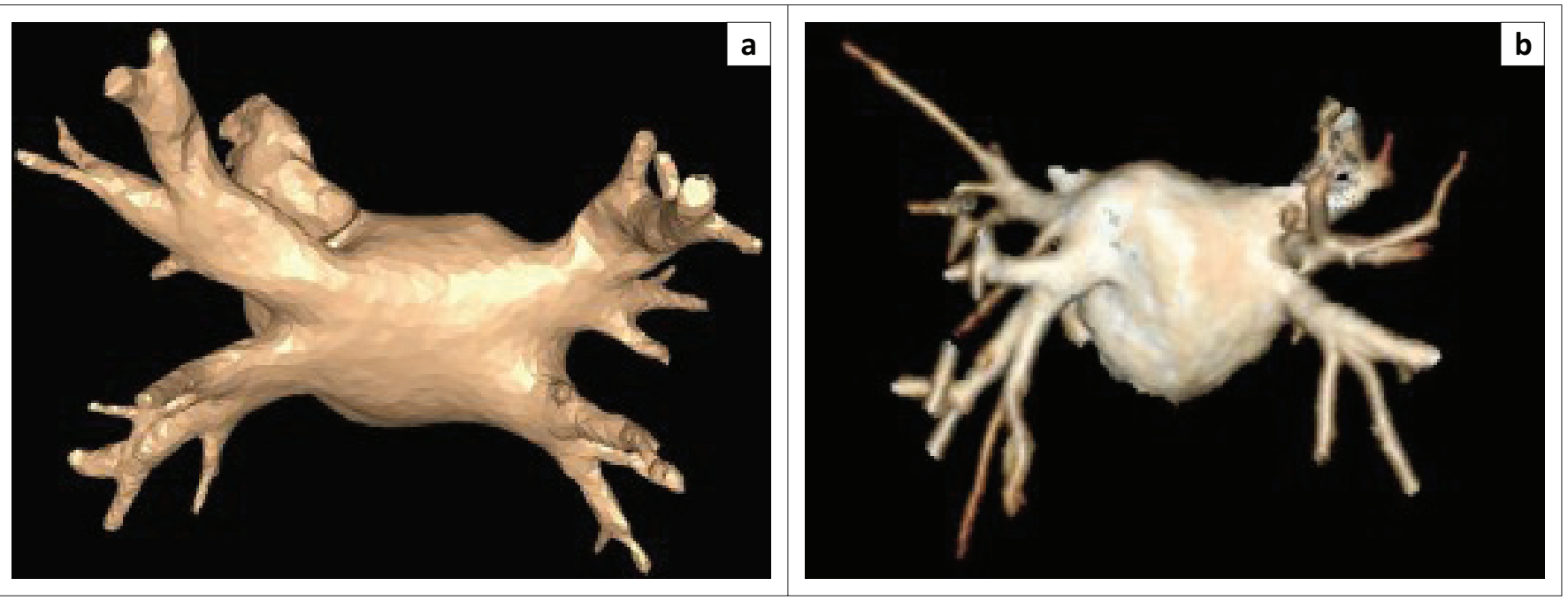

FIGURE 1: 3D reconstructed data from: (a) CTA and (b) MRA both show exquisite detail in the structure of the left atrium and pulmonary veins.

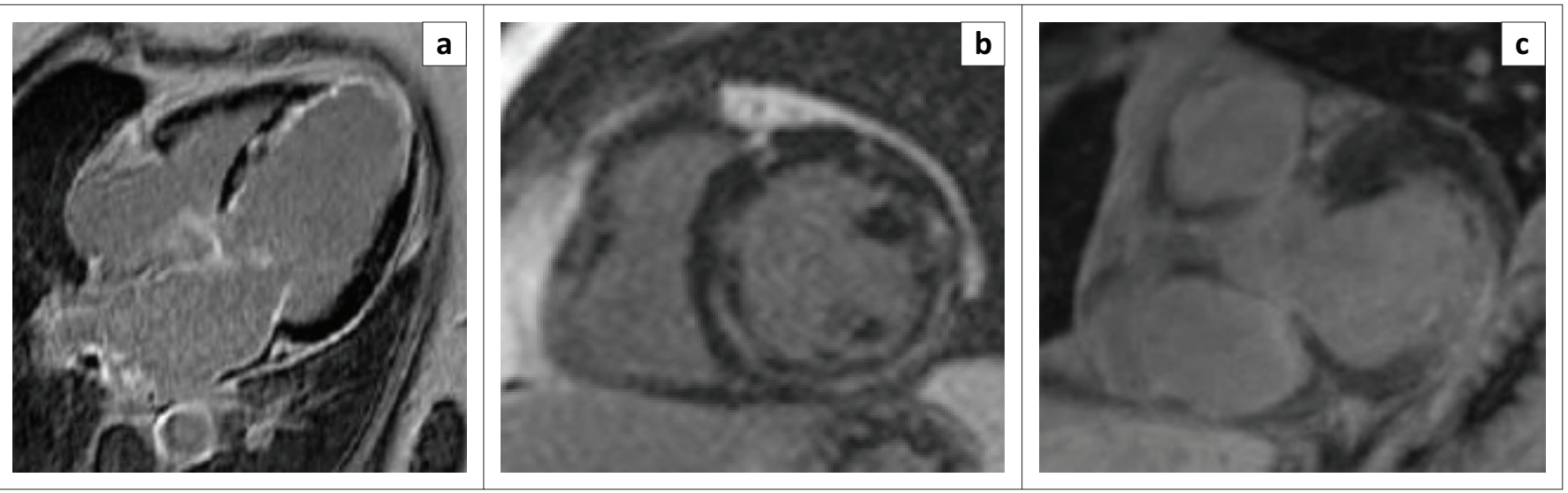

FIGURE 2: Late gadolinium enhancement showing scar patterns in different cardiomyopathies: (a) ischaemic cardiomyopathy, with subendocardial and transmural components; (b) cardiac sarcoid, with a patchy multifocal pattern; and (c) myocarditis, with a primarily subepicardial pattern. 


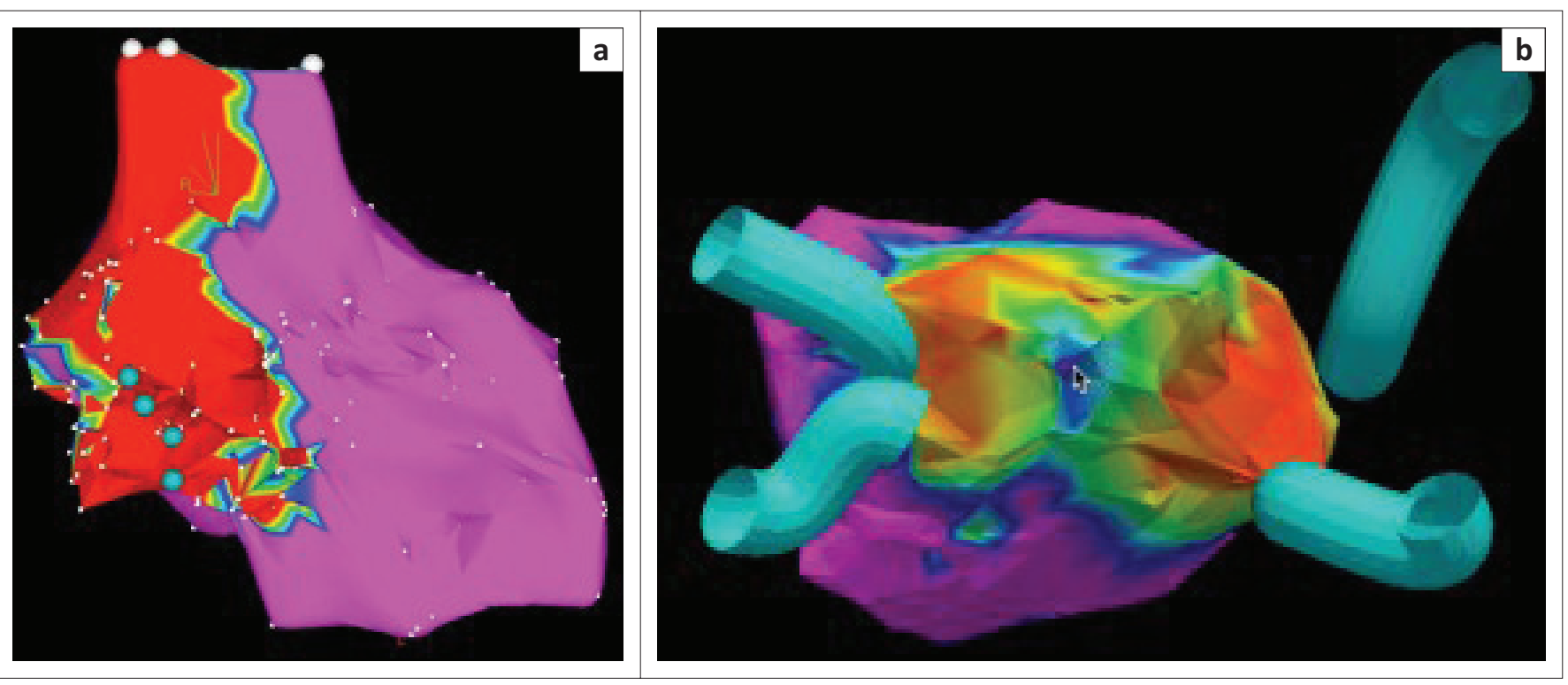

FIGURE 3: Electroanatomical maps (EAMs) of cardiac chambers: (a) EAM of the left ventricle. Red areas represent low voltage (scar) and purple areas represent normal voltage; (b) EAM of the left atrium, with tubular structures representing the pulmonary veins. Area of ablation shows low voltage (red) and non-ablated areas show normal voltage (purple).

electrical activity in the heart, as well as to guide cardiac ablation therapy. A specialised catheter is advanced into the heart, and its manually guided tip electrode is apposed against the myocardial surface to measure electrical activity. Areas of normal myocardium produce high electrical activity, and areas of scar produce low electrical activity. Systems have been developed to precisely track the position of the catheter tip in 3D space. A set of coils on the table or patches on the patient generate either a magnetic field or an electrical field, which is detected by special sensors at the tip of the intracardiac catheter, enabling determination of its location in $3 \mathrm{D}$ space. By localising in 3D space each measurement of cardiac electrical activity, 3D surface endocardial or epicardial maps of cardiac electrical activity are generated. An endocardial map of the left ventricle (LV) (Figure 3a) and of the left atrium (LA) (Figure 3b) are illustrated. Endocardial EAMs are generated by intracardiac catheters, whereas epicardial EAMs are generated by intrapericardial catheters or by catheters into epicardial coronary veins.

Although EAMs provide anatomic maps of cardiac electrical activity, they are quite crude, as they are generated typically from 100 to 300 catheter measurements (although mapping systems allow 1000 measurements or more). They offer a distorted image of cardiac chamber anatomy and are electrically undersampled. There has been a considerable effort to integrate the better anatomic and scar information provided by CT and MRI datasets into the 3D EAMs, in order to enhance procedural outcomes. There is generally good correspondence between spatial distribution of scar on EAMs and scar on MRI, with some mismatches owing to EAM undersampling or poor catheter contact (Figure 4). MRI offers precise assessment of transmural distribution of scar, and can easily distinguish between subendocardial scar and subepicardial scar. EAMs are less precise than MRI to determine transmural

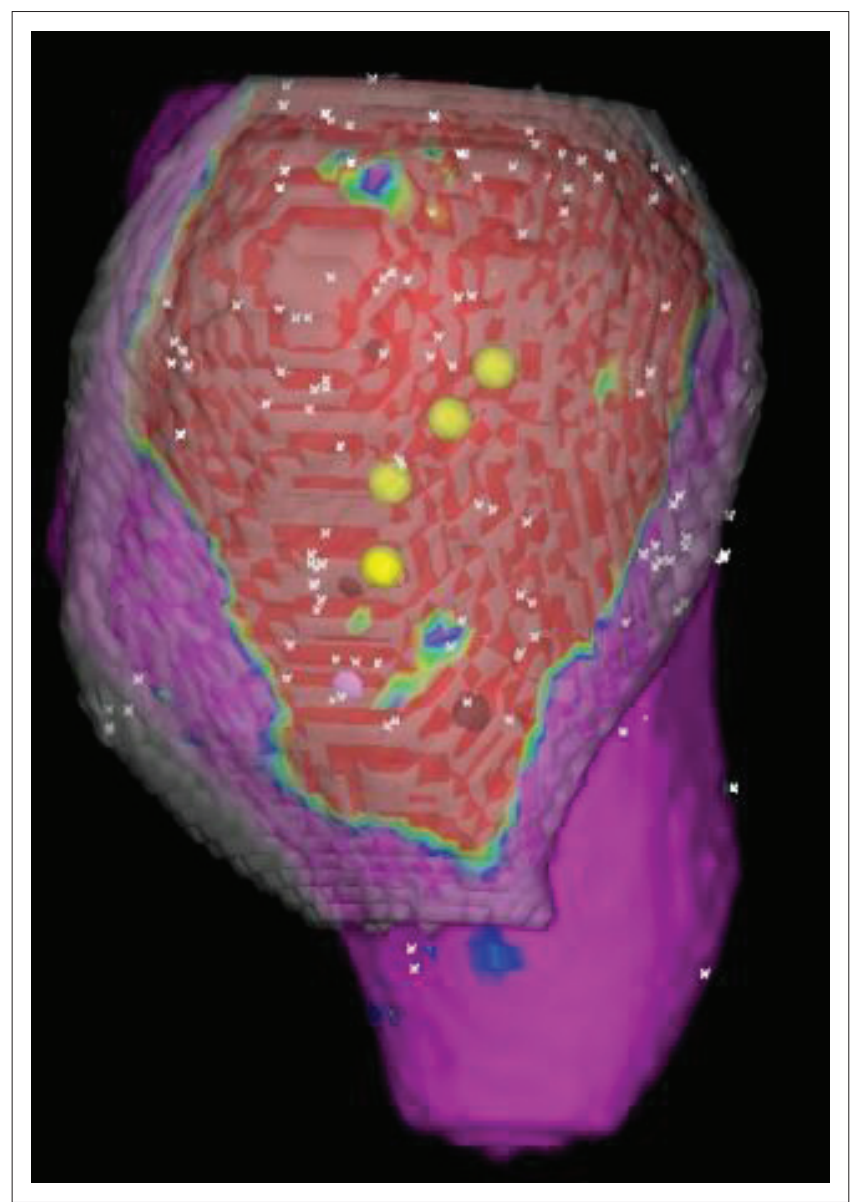

FIGURE 4: Registration of EAM and late gadolinium MRI. There is a slight mismatch on the left side of the image, where an area of normal voltage on EAM (purple) is superimposed on an area of scar on MRI (gray). This resulted from a gap in sampling on the EAM.

extent of scar. EAMs are very difficult to interpret when scar distribution is complex or patchy, which is often seen in non-ischaemic cardiomyopathy, whereas complex scar can be better assessed by MRI. 


\section{Image integration}

To integrate 3D data from CT or MRI with EAMs is a twostep process. The CT or MRI data must first be imported into the EAM system, and must then be registered to the patient's anatomy. For left atrial anatomy, a lasso catheter is systematically moved into the LA and pulmonary veins, rapidly creating a $3 \mathrm{D}$ point cloud map representing the patient's anatomy, which is then registered to the imported 3D CT or MRI surface anatomy. For LV anatomy, fiducials (mitral valve, aortic valve, LV apex) are identified by catheter and on the 3D CT or MRI data, and both are registered via these fiducials. Registration is then refined using surface-based algorithms, producing a registration error less than $3 \mathrm{~mm}$ in accuracy.

\section{Atrial fibrillation}

$\mathrm{AF}$ involves the chaotic electrical activity in the atria (350 bpm - $600 \mathrm{bpm}$ ) causing quiver instead of an organised contraction. It is caused by abnormal depolarisation of excitable myocardial tissue extending into the pulmonary veins. It is the most common arrhythmic cause for hospitalisation, causing a twofold increase in mortality and fivefold increase in strokes. Two common forms of therapy are medication, which has frequent toxicity and a high rate of failure, and ablation therapy by catheter, which involves the creation of linear thermal lesions to electrically isolate the different parts of the LA.

\section{Planning}

Imaging can help to plan left atrial ablation therapy. It is used (1) in defining the anatomy for proper ablation therapy, (2) to identify potential contraindications to ablation, (3) to identify the relationship between the LA and surrounding structures that can be injured by ablation, and (4) to identify which subjects are more at risk of developing AF.

Electrical isolation of the pulmonary veins from the LA requires proper planning. The size of the ostia of the pulmonary veins will guide proper catheter selection. The pattern of the pulmonary veins and variant anatomy will affect catheter manipulation and delivery of ablation lesions. The presence of a thrombus in the left atrial appendage can be readily identified by imaging and is a contraindication to ablation.

Structures surrounding the LA can suffer thermal injury from the ablation lesions. The oesophagus courses along the posterior wall of the LA and comes into close contact with the wall, separated only by a thin layer of fat. Identifying by imaging the path of the oesophagus with respect to the LA will determine which ablation lesions are at greater risk of causing potentially lethal thermal injury to the oesophagus, and requires ablation with reduced energy in these areas. The right phrenic nerve travels close to the ostia of the pulmonary veins. Identifying by imaging the precise path of the right phrenic nerve helps to prevent thermal injury to the nerve and permanent phrenic palsy.
Subjects with dilated LA are associated with an increased risk of recurrence of AF after ablation. Imaging allows precise measurement of LA volume. More recently (and more controversially), the degree and extent of scarring of the LA owing to chronic AF might be able to predict the risk of recurrence of AF following ablation. ${ }^{1}$ Assessment of LA volume and LA scar by imaging could help to select the appropriate candidates for ablation.

\section{Guidance}

Imaging can guide left atrial ablation therapy. The LA and pulmonary veins have a complex and variable anatomical pattern, and the ablation procedure involves the creation of linear lesions to electrically isolate the different parts of the LA. However, ablation catheters can only perform punctiform lesions, and linear lesions are actually the result of several collinear punctiform lesions without gaps between them (Figure 5). Imaging guidance enables precise location of the ablation lesions, improving proper electrical isolation without gaps at optimal locations within the LA. This leads to improvement in clinical outcome ${ }^{2}$, decrease in procedure duration, and decrease in fluoroscopy time. Modern LA ablation techniques involve guidance under virtual reality. The 3D anatomical information from CT or MRI is imported into the EAM software and registered to the patient. The procedure is then entirely virtually guided by the imported LA anatomy (Figure 6a). In addition, anatomy of the oesophagus and the phrenic nerves is also used to guide the ablation (Figure 6b), and minimise the risk of thermal injury to these structures.

Intraprocedural imaging by intracardiac echocardiography (ICE) is becoming integrated into EAM systems. ICE involves a steerable intravascular catheter using a 64-element vector phased array, with tissue penetration of $16 \mathrm{~cm}$. This technique improves real-time catheter navigation, real-time $3 \mathrm{D}$ reconstruction of LA anatomy, helps to guide transseptal

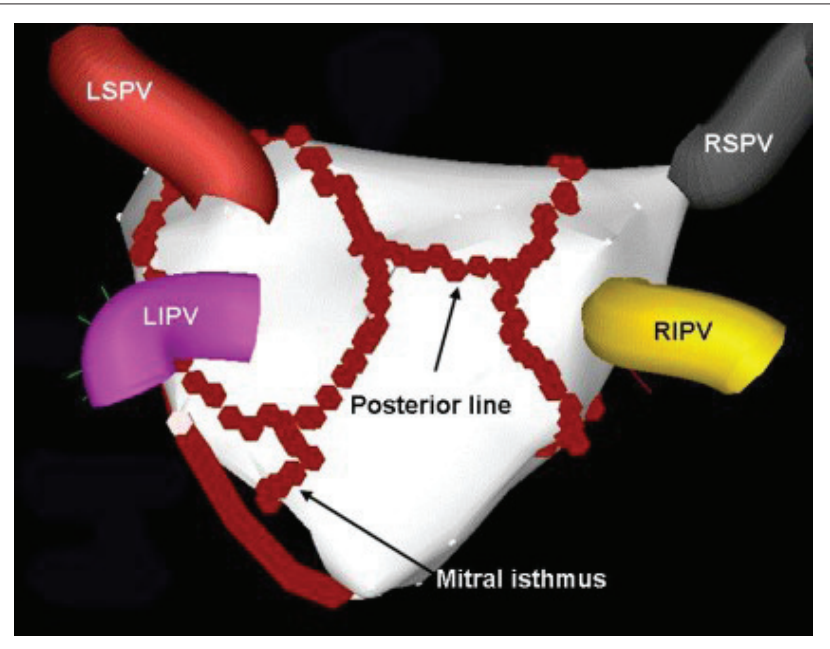

PV, pulmonary vein; LSPV, left superior PV; RSPV, right superior PV; LIPV, left inferior PV; RIPV, right inferior PV.

FIGURE 5: Illustration of ablation lines electrically isolating the different parts of the left atrium. Each line is made up of collinear punctiform ablation lesions without gaps. 


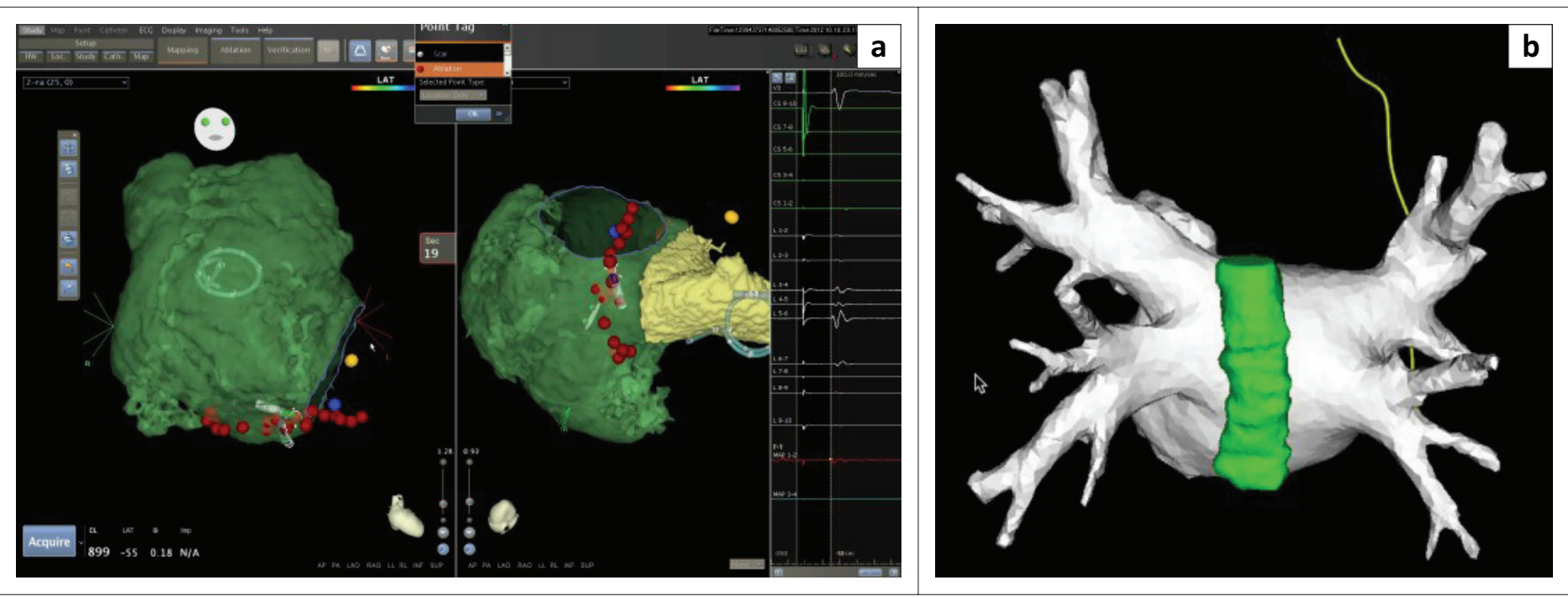

FIGURE 6: An ablation procedure guided by CT data: (a) The ablation catheter is visualised in real-time and ablation lesions are represented by red dots; (b) Besides the structure of the left atrium and pulmonary veins, surrounding structures such as the oesophagus (green) and phrenic nerve (yellow) can be used to guide ablation therapy.

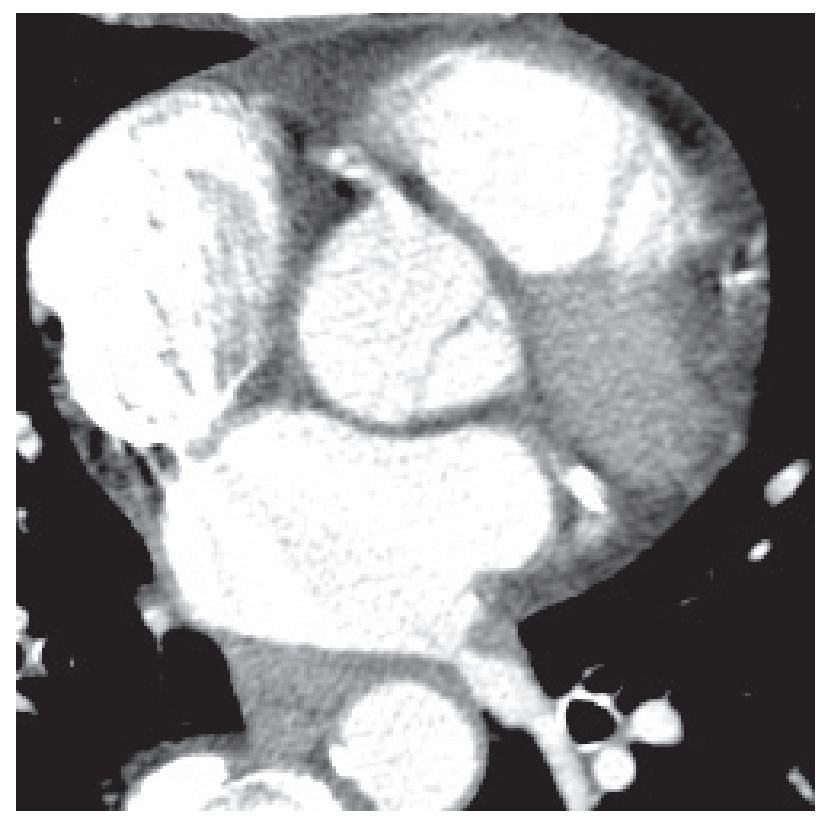

FIGURE 7: CTA shows significant narrowing of the ostium of the left inferior pulmonary vein, a complication of ostial ablation therapy.

puncture to access the LA from the right atrium (RA), visualise the pulmonary vein ostia, visualise the oesophagus to avoid collateral damage, and helps to recognise complications such as perforations. ICE with acoustic radiation force imaging ${ }^{3}$ can assess tissue elasticity and directly visualise ablation lesions, which are stiffer than normal portions of the LA, and can potentially identify gaps.

\section{Post procedure}

Imaging can identify complications of ablation therapy, such as pulmonary vein stenosis (Figure 7), which used to happen in up to $42 \%$ of procedures (although this has decreased to $1 \%$ with techniques ablating further away from the pulmonary veins ostia ${ }^{4}$ ), as well as identify other complications, such as thrombosis, dissection, pulmonary infarction or atrio-oesophageal fistula. More recently, MRI has been used to assess results of LA ablation therapy. ${ }^{5}$ The ablation lesions behave similarly to myocardial scar on MRI, but with much longer wash-in and wash-out kinetics. ${ }^{6}$ They show as bright signal on MRI days to months after the procedure (Figure $8 \mathrm{a}, \mathrm{b}$ ). In about $35 \%$ of cases, AF recurs weeks or months after the ablation procedure, owing to development of gaps in the linear ablation lesions. ${ }^{7}$ It is now possible to identify by imaging these developing gaps in the ablation lines causing failure of therapy, and perform a very targeted redo procedure to eliminate those gaps.

\section{Ventricular tachycardia}

Cardiomyopathies, which are diseases involving a deterioration of the function of the myocardium, increase the risk of arrhythmia and sudden cardiac death. Cardiomyopathies often present with myocardial scar, which acts as an arrhythmogenic substrate, and surviving muscle bundles within scar are probably responsible for arrhythmia, as they create alternate slow pathways for cardiac conduction. ${ }^{8}$ Imaging can readily identify several of these pathologies. Ischaemic cardiomyopathy involves subendocardial scar of variable transmurality, along a vascular territory, accompanied by regional wall motion abnormalities and anatomical remodeling (Figure 2a). Hypertrophic cardiomyopathy involves wall thickening, with areas of thickening showing diffuse patchy scar. Other non-ischaemic cardiomyopathy can reveal multifocal scar (e.g. cardiac sarcoid, Figure 2b) or subepicardial scar (e.g. myocarditis, Figure 2c). Therapy for cardiomyopathy includes the use of medication, the implantation of intracardiac defibrillators, or ablation therapy.

Cardiac ablation therapy via catheter can be used to destroy the abnormal conduction pathways. Unlike left atrial ablation therapy, where ablation lines are created to electrically isolate different parts of the LA, ablation lesions in the LV are more clustered, and their purpose is to homogenise the ventricular substrate, to transform mixed scar into pure scar.

Risk stratification for ischaemic cardiomyopathy to determine which patients require an implantable cardiac 


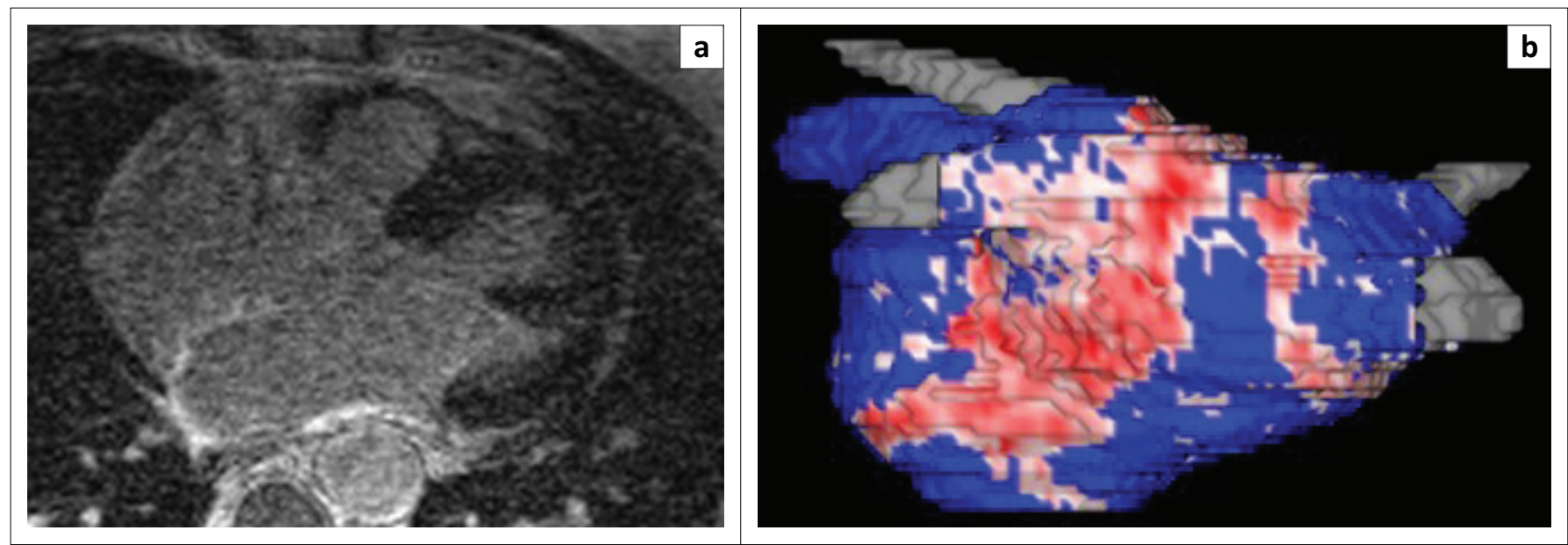

FIGURE 8: Late gadolinium enhancement (a) of the left atrium post ablation. Ablation lesions present as bright signal on an otherwise dark myocardium; (b) 3D map representation of the scar (red) distribution.

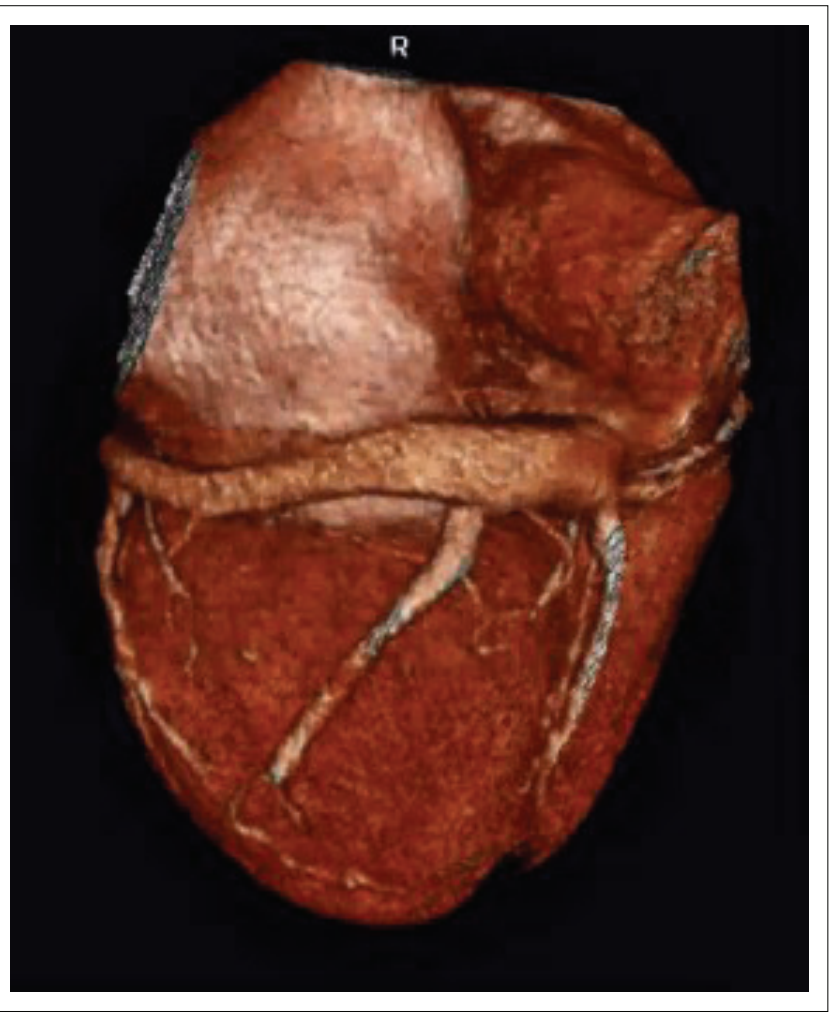

FIGURE 9: Surface-rendered CTA demonstrates the path of the coronary sinus and coronary veins, for planning of implantation of a biventricular pacemaker or ICD.

defibrillator (ICD) currently involves assessment of cardiac function. Patients with depressed cardiac functions and left ventricular ejection fraction (LVEF) $<35 \%$, will typically require an ICD. But a large proportion of patients with ICDs never experience an ICD discharge (and therefore do not really need one), and some patients who are not expected to need an ICD present with arrhythmia or sudden cardiac death. MRI can assess scar distribution and heterogeneity and can offer better risk stratification. Scar involving $25 \%-75 \%$ of the wall thickness is predictive of inducible ventricular tachycardia. Heterogeneous scar showing areas of intermediate signal (gray zone, indicating scar mixed with normal myocardium) is also predictive of ventricular arrhythmia.

\section{Planning}

Imaging can help to plan for therapy in cardiomyopathy. Visualising the anatomy of the coronary sinus and coronary veins is useful prior to implantation of a biventricular pacemaker or ICD. In these devices, a third lead is inserted via the coronary sinus and follows an epicardial vein along the surface of the LV. Precise knowledge of the course and size of the epicardial veins helps optimal placement of this third lead (Figure 9). Scar imaging helps to avoid placement of leads in areas of non-viable myocardium which would not be responsive to pacing, and functional imaging can identify areas of latest activation which are optimal for lead placement.

The same anatomy is also useful to guide epicardial ablation therapy via the coronary veins, and helps to achieve a higher success rate of ablation of some patterns of epicardial scar. Visualising the anatomy of the coronary arteries is useful in epicardial ablation, as coronary arteries are prone to damage by thermal energy from the ablation. One should avoid ablating within $5 \mathrm{~mm}-10 \mathrm{~mm}$ from a coronary artery to prevent vascular damage.

Determining the thickness of epicardial fat by imaging is also useful for planning epicardial ablation. Unlike endocardial ablation, where the catheter is in close contact with the myocardium (the endocardium is a very thin layer), in epicardial ablation a layer of epicardial fat of variable thickness separates the myocardium from the catheter. Epicardial fat $>3 \mathrm{~mm}$ thick attenuates amplitudes of epicardial electrograms ${ }^{9}$, and thick fat can mimic scar. In those cases, fragmented electrograms can help to distinguish scar from thick epicardial fat. Furthermore, the ablation procedure can be completely ineffective in areas of very thick epicardial fat $(>10 \mathrm{~mm})$.

Imaging scar location and distribution is important to plan ventricular ablation therapy. Critical sites for arrhythmia (target sites for ablation) are typically located in areas of scar demonstrated by MRI. ${ }^{10}$ Ablation can target the precise chambers and wall containing scar on MRI, saving procedure time and improving outcomes. The distribution of scar across 
the LV wall can determine the approach for successful ablation. An endocardial approach will be successful for subendocardial scar, and an epicardial approach will be successful for subepicardial scar. If the scar is predominantly intramural, such as in non-ischaemic cardiomyopathy, both approaches might fail.

Viable but denervated myocardium around scar is prone to arrhythmias, although the underlying mechanism for arrhythmias is not clear. ${ }^{11}$ After a myocardial infarct, the sympathetic denervation defect is usually larger than the area of scar, and ablation of this viable but denervated myocardium can successfully eliminate critical foci for arrhythmia. Areas of denervation can be demonstrated by SPECT imaging using meta-iodobenzylguanidine (MIBG), a norepinephrine analogue, or by PET imaging using hydroxyephedrine (HED), an epinephrine analogue. Correlation of extent of denervation with extent of scar on MRI can identify additional target sites for ablation.

\section{Guidance}

EAMs have been used to guide ventricular ablation therapy with $50 \%-80 \%$ success and $40 \%$ recurrence post ablation. ${ }^{12}$ Imaging could improve these outcomes. Intracardiac echography (ICE) can guide ablation during the procedure. The ICE catheter is inserted in the right heart and produces ECG-gated 90-degree sector images to generate 3D volumes of the heart. This can improve registration with CT and MRI images, ${ }^{13}$ identify complications such as thrombus, and identify scar tissue by wall thinning or increased echo density. This can also improve control of the tip of the ablation catheter, to target arrhythmias in difficult locations such as papillary muscles. ${ }^{14}$

Real-time MRI is also used to guide ablation therapy. ${ }^{15}$ Advantages are absence of ionising radiation, real-time creation of lesions, real-time assessment of scar and monitoring of lesion temperature. Disadvantages are lack of Food and Drug Administration (FDA) approval, and a significant change in workflow and financial investment. The main technical problems have, however, been solved. Specialised ablation catheters have been developed which are actively trackable under MRI. Thermographic pulse sequences have been developed that can assess the extent of thermal injury from each ablation lesion. Techniques have been developed to correct ECG signal distortion inside the scanner. MRI-guided ablation could have a great future, but currently remains a purely experimental technique.

\section{Post procedure}

MRI can be used to assess the location of the ventricular ablation lesions. The enhancement kinetics of these ablation lesions in the LV require a very long delay for LGE imaging $\left(90 \mathrm{~min}^{6}\right)$, which is not practical. More recent techniques such as T1 mapping, which do not depend on IV contrast kinetics, have been developed to acutely visualise core ablation lesions and surrounding oedema in the LV. ${ }^{16}$ Although clinical outcome determines the success of the ablation procedure, imaging of ventricular ablation lesions can help to identify location of suboptimal ablation lesions if arrhythmia recurs after ablation.

\section{Conclusion}

Imaging has numerous uses in clinical cardiac electrophysiology. It helps to diagnose cardiomyopathy; it can assess cardiac anatomy, function and scar; it helps to plan and guide interventions such as device placement or ablation therapy; and it can be used to assess the result and complications of these procedures.

Although much progress has been made to image arrhythmogenic substrate and integrate the imaging data into mapping and ablation systems, there is plenty of scope for improvement. The spatial resolution of images of scar needs improvement, registration of imported 3D structures with the patient is far from perfect, and in vivo imaging with ICE or MRI needs further development. ${ }^{17}$ Cost effectiveness still limits availability, and superior outcomes have yet to be demonstrated.

\section{Acknowledgements Competing interests}

The author declares that he has no financial or personal relationships which may have inappropriately influenced him in writing this article.

\section{References}

1. Oakes RS, Badger TJ, Kholmovski EG, et al. Detection and quantification of left atrial structural remodeling with delayed-enhancement magnetic resonance imaging in patients with atrial fibrillation. Circulation. 2009;119:1758-1767. http://dx.doi.org/10.1161/CIRCULATIONAHA.108.811877

2. Bertaglia $E$, Bella PD, Tondo $C$, et al. Image integration increases efficacy of paroxysmal atrial fibrillation catheter ablation: Results from the CartoMerge
Italian Registry. Europace. 2009;11:1004-1010. http://dx.doi.org/10.1093/ Italian Registry.
europace/eup152

3. Eyerly SA, Hsu SJ, Agashe SH, et al. Intracardiac acoustic radiation force impulse imaging: A novel imaging method for intraprocedural evaluation of radiofrequency ablation lesions. Heart Rhythm. 2012;9:1855-1862. http://dx.doi.org/10.1016/j. hrthm.2012.07.003

4. Holmes DR, Monahan KH, Packer D. Pulmonary vein stenosis complicating ablation for atrial fibrillation: Clinical spectrum and interventional considerations. J Am Coll Cardiol Interv. 2009;2(4):267-276. http://dx.doi.org/10.1016/j. jcin.2008.12.014

5. Peters DC, Wylie JV, Hauser TH, et al. Detection of pulmonary vein and left atria scar after catheter ablation with three-dimensional navigator-gated delayed enhancement MR imaging: Initial experience. Radiology. 2007;243:690-695. http://dx.doi.org/10.1148/radiol.2433060417

6. Dickfeld T, Kato R, Zviman M, et al. Characterization of radiofrequency ablation lesions with gadolinium-enhanced cardiovascular magnetic resonance imaging. J Am Coll Cardiol. 2006;47:370-378. http://dx.doi.org/10.1016/j.jacc.2005.07.070

7. Badger TJ, Daccarett M, Akoum NW, et al. Evaluation of left atrial lesions after initial and repeat atrial fibrillation ablation: Lessons learned from delayed enhancement MRI in repeat ablation procedures. Circ Arrhythm Electrophysiol. 2010;3:249-259. http://dx.doi.org/10.1161/CIRCEP.109.868356

8. Bolick DR, Hackel DB, Reimer KA, et al. Quantitative analysis of myocardial infarct structure in patients with ventricular tachycardia. Circulation. 1986;74: 1266-1279. http://dx.doi.org/10.1161/01.CIR.74.6.1266

9. Desjardins B, Morady F, Bogun F. Effect of epicardial fat on electroanatomical mapping and epicardial catheter ablation. J Am Coll Cardiol. 2010;56:1320-1327. http://dx.doi.org/10.1016/j.jacc.2010.04.054

10. Bogun FM, Desjardins B, Good E, et al. Delayed-enhanced magnetic resonance imaging in nonischemic cardiomyopathy: Utility for identifying the ventricular arrhythmia substrate. J Am Coll Cardiol. 2009;53:1138-1145. http://dx.doi. org/10.1016/j.jacc.2008.11.052

11. Simoes MV, Barthel P, Matsunari I, et al. Presence of sympathetically denervated but viable myocardium and its electrophysiologic correlates after early revascularised, acute myocardial infarction. Eur Heart J. 2004;25:551-557. http://dx.doi.org/10.1016/j.ehj.2004.02.016 
12. Tanner H, Hindricks G, Volkmer M, et al. Catheter ablation of recurrent scar-related ventricular tachycardia using electroanatomical mapping and irrigated ablation technology: Results of the prospective multicenter Euro-VT-study. J Cardiovasc

3. Bunch TJ, Weiss JP, Crandall BG, et al. Image integration using intracardiac ultrasound and $3 \mathrm{D}$ reconstruction for scar mapping and ablation of ventricula tachycardia. J Cardiovasc Electrophysiol. 2010;21:678-684. http://dx.doi. org/10.1111/j.1540-8167.2009.01680.x

14. Good E, Desjardins B, Jongnarangsin $K$, et al. Ventricular arrhythmias originating from a papillary muscle in patients without prior infarction: A comparison with fascicular arrhythmias. Heart Rhythm. 2008:5:1530-1537. http://dx.doi. org/10.1016/j.hrthm.2008.08.032
15. Nazarian S, Bluemke DA, Lardo AC, et al. Magnetic resonance assessment of the substrate for inducible ventricular tachycardia in nonischemic cardiomyopathy. Circulation. 2005;112:2821-2825. http://dx.doi.org/10.1161/CIRCULATIONAHA. 105.549659

16. Ranjan R, Kholmovski E, Silvernagel J, et al. Acute assessment of radiofrequency ablation lesions in MRI without the use of gadolinium based contrast. J Am Coll Cardiol. 2014;63(12 suppl.):A389. http://dx.doi.org/10.1016/s0735-1097(14) 60389-2

17. Njeim M, Desjardins B, Bogun F. Multimodality imaging for guiding electrophysiology ablation procedures. JACC Imaging. 2016;9(7):873-886. 